\title{
ERCP: does patient position count?
}

\section{(ㄷ)(이우}

\author{
Authors \\ Amit Maydeo, Gaurav Kumar Patil \\ Institution \\ Baldota Institute of Digestive Sciences (BIDS), Global \\ Hospital, Parel, Mumbai 400012, India \\ Bibliography \\ DOI https://doi.org/10.1055/a-0732-5193 | \\ Endoscopy International Open 2018; 06: E1302-E1303 \\ (c) Georg Thieme Verlag KG Stuttgart · New York \\ ISSN 2364-3722
}

Corresponding author

Amit Maydeo, MD, Baldota Institute of Digestive Sciences

(BIDS), Global Hospital, Parel, Mumbai 400012, India

Fax: +91-22-56642140

amitmaydeo@gmail.com
ERCP has come a long way since the first report of ampulla of Vater cannulation by McCune et al. in 1968 [1]. In the initial period since its description, ERCP was always performed with patients lying in the left lateral position, and as it became established, endoscopists carried out this procedure with patients lying in the prone position. This position was selected because of the ease of visualization and cannulation of the ampulla of Vater. Earlier textbooks and videos on advanced endoscopy recommended that the patient be placed with the left arm lying on the back and with subsequent rotation into the prone position. This required turning the patient's body using triangular wedges or cylindrical pillows [2]. However, this semi-prone position was sometimes cumbersome and uncomfortable for patients who had abdominal distension, ascites, recent abdominal or neck surgery, indwelling percutaneous tubes or catheters, endotracheal anesthesia, and those who were morbidly obese. In addition, chest rolls could prevent compromise of chest expansion with the patient in the prone position.

The supine position was usually reserved for patients undergoing complex procedures under general anesthesia with intubation or for intensive care patients. Theoretically, the supine position had some distinct advantages over the prone position: (1) It permitted excellent radiological documentation and was more comfortable for the patient. (2) It enabled easier manipulation of the patient on the fluoroscopy table for better visualization in various positions, especially when a rotatable C-Arm was used. (3) The supine position was best suited for visualization of the hepatic hilum and for pancreatic endotherapy with a true anatomical visualization instead of the inverted image in the prone position. (4) The supine position gave easier access to the patient's respiratory system, the potential risk of aspiration being obviated by continuous salivary aspiration by assisting staff.
In fact, many leading endoscopy centers in the world did perform ERCP in the supine position using total intravenous anesthesia (TIVA) without any significant incidences of aspiration or respiratory distress.

Until 2008, there was no published data on the impact of patient positioning on the safety and outcomes of ERCP. In this issue of Endoscopy International Open, in the study "Comparison of outcomes for supine vs prone position ERCP: a systematic review and meta-analysis" [3], the authors have compared the technical success and safety outcomes of prone vs supine position in ERCP. The pooled technical success was shown to be slightly higher with prone positioning, and complications were on the lower side; however, there was significant heterogeneity in the studies in terms of sample size and also the nature of the studies.

In a retrospective analysis published in 2008, Ferreira and Baron [4] compared the safety and efficacy of ERCP prone and supine positions. All patients in whom ERCP could not be performed in the conventional prone position were operated in the supine position. The authors observed that there were no differences between the two groups with respect to procedural time, American Society of Anesthesiologists (ASA) scores or adverse cardiopulmonary events. On subgroup analysis, the results of pre-cut sphincterotomy, selective ductal access, and complications were similar in the two groups. Thus, the authors concluded that an ERCP with the patient in the supine position could be safely and effectively performed if specifically warranted by clinical circumstances.

In a prospective randomized study published by Tringali et al. in 2008 [5], the authors reported the outcomes in 120 patients who underwent ERCP in the supine or prone positions and using conscious sedation. They found no differences in the time to reach the major papilla, to achieve a successful cannulation of the desired duct, or in total duration of the procedure; 
both their groups were comparable in terms of procedural success, technical difficulty, and complications.

It has been the experience of numerous ERCP experts that the success of selective cannulation depends predominantly upon many technical factors such as proper positioning of the ampulla of Vater in the right axis, proper orientation of the sphincterotome, using the right guide/glide wire, gentle handling of the ampulla during cannulation, etc. These factors can also be easily satisfied in the supine position with some simple steps of scope tip and shaft manipulation. In addition, if the endoscopist ensures that the stomach is emptied as soon as the duodenoscope enters it and intermittent pharyngeal suction is performed during the procedure, the theoretical fear of aspiration is prevented.

The paper by Terruzzi et al. [6], which has been cited by the authors, also has its own limitations. The number of patients in that study was small and enrollment was stopped after the planned interim analysis because it became clear that the prone position was overwhelmingly superior to the supine position in terms of both safety and difficulty of cannulation. This could be because the endoscopists were not used to operating on patients in the supine position thereby making orientation of the ampulla difficult for cannulation and only patients with a biliary indication were included.

In fact, most of the studies quoted by the authors have these limitations and there is a lack of power in terms of sample size to exclude definitively type I and II statistical errors.

Because ERCP is currently largely therapeutic, it requires a dedicated infrastructure and a multidisciplinary team with adequately trained personnel. In the modern world of rapidly advancing technology, endoscopes have also evolved, and the optical system/field of vision of modern duodenoscopes is far superior to the fiberoptic duodenoscope used by Oi [7]. With improved scope optics, field of vision, range of movement of the distal bending section, and variety of user friendly ERCP accessories, it is quite easy to accomplish a successful ERCP even in the supine position. In fact, out of all of the factors leading to the success of therapeutic ERCP, patient position is probably the least contributory.

To reach a meaningful conclusion on whether patient position or its associated complications count towards ERCP success, we need further well outlined prospective studies with an adequate sample size. Until such studies are conducted, it will continue to be debated whether patient position really counts while performing this immensely popular procedure.

\section{Competing interests}

None

\section{References}

[1] McCune WS, Shorb PE, Moscovitz H. Endoscopic cannulation of the ampulla of Vater: a preliminary report. Ann Surg 1968; 167: 752 - 756

[2] Cotton PB, Williams C. ERCP: diagnostic technique. In: Cotton PB, Williams $C$, eds. Practical gastrointestinal endoscopy. 4th edn. Cambridge, MA: Blackwell Science; 1996: 105-138

[3] Mashiana H, Jayaraj M, Mohan B et al. Comparison of outcomes for supine vs prone position ERCP: A systematic review and meta-analysis. Endosc Int Open 2018; 06: E1296-E1301

[4] Ferreira LEVVC, Baron TH. Comparison of safety and efficacy of ERCP performed with the patient in supine and prone positions. Gastrointest Endosc 2008; 67: 1037 - 1043

[5] Tringali A, Mutignani M, Milano A et al. No difference between supine and prone position for ERCP in conscious sedated patients: a prospective randomized study. Endoscopy 2008; 40: 93 - 97

[6] Terruzzi V, Radaelli F, Meucci G et al. Is the supine position as safe and effective as the prone position for endoscopic retrograde cholangiopancreatography? A prospective randomized study Endoscopy 2005; 37: $1211-1214$

[7] Oi I. Fiberduodenoscopy and endoscopic pancreatocholangiography. Gastrointest Endosc 1970; 17: 59-62 